\title{
Vakuumversiegelung bei Fasciitis necroticans vor Spalthautver- pflanzung - nicht nur eine Ultima Ratio?
}

\begin{abstract}
Zusammenfassung. Es wird über einen 72-jährigen Patienten berichtet, der aufgrund einer Fasciitis necroticans am rechten Oberschenkel einen großflächigen Defekt bis auf die Muskulatur entwickelte. Es erwies sich als unmöglich, den Defekt mittels einer stufenweisen Dehnungsplastik zu verschließen. Eine Spalthauttransplantation war erst erfolgreich, nachdem der Wundgrund mit Hilfe einer Vakuumversiegelung für zehn Tage konditioniert worden war.
\end{abstract}

Vacuum Sealing of Fasciitis necroticans Wounds Before Split Skin Grafting - Not Only a Last Resort? We report on a 72-year old patient who developed a large defect on his right thigh due to fasciitis necroticans. The defect extended to the muscles and could not be closed by means of a stepwise advancement of the wound edges. Split skin grafting was only successful after the wound had been conditioned with vacuum sealing for ten days.

\section{Einleitung}

Die Behandlung von Verletzungen und Wunden ist uraltes Wissensgut der Menschheit. Galen schlug im zweiten Jahrhundert erstmals das Konzept der primären und der sekundären Wundheilung vor. Voraussetzung für eine Sanatio per primam intentionem ist eine spannungsfreie Adaptation der Wundränder. Bei Problemwunden mit auseinanderliegenden (gequetschten oder nekrotischen) Wundrändern oder einer Wundinfektion erfolgt dagegen eine verzögerte sekundäre Wundheilung. Der Gewebedefekt wird dabei zunächst durch Granulationsgewebe aufgefüllt. Dieses epithelisiert vom Rand her unter ausgedehnter Narbenbildung [1].

Mit diesem Fallbericht möchten wir die Methode der Vakuumversiegelung von Problemwunden vorstellen, die sich auch zur Vorbereitung von Spalthauttransplantationen eignet. Die Technik der Vakuumversiegelung wurde 1993 erstmals von Fleischmann beschrieben [2]. In der Unfallchirurgie und der plastischen Chirurgie hat die Vakuumversiegelung bereits ih-

Akt Dermatol 2001; 27: 79-82

(c) Georg Thieme Verlag Stuttgart $\cdot$ New York ISSN 0340-2541

\author{
A. Frank, J.-M. Pönnighaus, L. Kowalzick \\ Klinik für Hautkrankheiten und Allergologie \\ der Vogtland-Klinikum Plauen $\mathrm{GmbH}$ \\ (Chefarzt: Priv.-Doz. Dr. med. L. Kowalzick)
}

ren festen Platz eingenommen, während sie in der operativen Dermatologie noch wenig verbreitet ist [3].

\section{Kasuistik \\ Anamnese}

Der 72-jährige Patient wurde uns von einer chirurgischen Klinik unter dem Verdacht einer blasenbildenden Dermatose verlegt. Bei Übernahme des Patienten war dieser ansprechbar aber desorientiert. Fremdanamnestisch war zu erfahren, dass seit ca. 10 Tagen Schmerzen im rechten Bein bestünden mit zunehmender Verschlechterung des Allgemeinzustandes. Ein Trauma war nicht eruierbar. Seit ca. 10 Jahren wurde der Patient wegen eines Diabetes mellitus mit Sulfonylharnstoff behandelt.

\section{Hautbefund}

Der rechte Oberschenkel war geschwollen und gerötet. Im lateralen Bereich fand sich eine ca. $20 \times 10 \mathrm{~cm}$ große schwarze Nekrose mit randständigen dünnhäutigen Blasen. Im gesamten ventrolateralen Oberschenkelbereich war ein deutliches Knistern zu tasten und zu hören.

\section{Allgemeinbefund}

RR 100/65 mmHg, Temp. rektal 37,4 ${ }^{\circ} \mathrm{C}$, schlechter Allgemeinzustand mit deutlichen Zeichen der Exsikkose, normaler Ernährungszustand.

Labor (SI)

Auffällig waren: Hb: 6,5 mmol/l, Hk: 0,31 l/l, GOT: 1,08 $\mu \mathrm{mol} / \mathrm{sl}$, Ca: $1,88 \mathrm{mmol} / \mathrm{l}$, K: 3,36 mmol/l, CrP: $266,2 \mathrm{mg} / \mathrm{l}$, BSG: $110 /$ >120, BZ: $8,1 \mathrm{mmol} / \mathrm{l}$, HBA1c: $9,1 \%$

Wundabstrich: E. coli, Klebsiella oxytoca, Enterokokken.

Röntgenthorax und Ruhe-EKG

Unauffällig. 


\section{Histologie}

Komplette Fasziennekrose mit granulozytärer Entzündung. Der Befund passt zu einer Gangrän.

\section{Diagnose}

Fasciitis necroticans.

\section{Therapie und Verlauf}

Noch am Aufnahmetag führten wir im lateralen Bereich des rechten Oberschenkels eine großzügige Spaltung und ausgedehnte Nekrosektomie bis auf die Muskulatur durch. Es entleerte sich ca. 1 Liter jauchigstinkende Flüssigkeit mit deutlich sichtbaren Gasbläschen (Abb.1). In der Folgezeit waren mehrere Redébridements notwendig. Es erfolgten sowohl lokale (Septopal-Ketten ${ }^{\circledR}$ ) als auch systemische antibiotische Therapien (gemäß Resistogramm). Begleitend zu diesen Maßnahmen mussten wegen eines starkem Hb-Abfalls 2 Ery-Konzentrate verabreicht und wegen der ausgeprägten Exsikkose mit Elektrolytverschiebung eine Infusionstherapie durchgeführt werden. Unter dieser Therapie besserten sich sowohl der Allgemeinzustand des Patienten als auch der Lokalbefund kontinuierlich. Es wurde zunächst versucht, den zirka $15 \times 35 \mathrm{~cm}$ großen Defekt durch eine stufenweise Dehnungsplastik zu verkleinern. Dies erwies sich jedoch als wenig erfolgreich, da die Fäden immer wieder - aufgrund der gewölbten Oberfläche - in die Muskulatur einschnitten. Das Instrumentarium für ein in der Literatur beschriebenes Verfahren zur schrittweisen Wundrandannäherung (Sure-Closure Skin Stretching System ${ }^{\circledR}$ ) war in Deutschland nicht erhältlich $[4,5]$. Nach ca. 4 Wochen konnten mit Alginatverbänden jedoch saubere, reizlose Wundverhältnisse erreicht werden, so dass nunmehr eine Defektdeckung mittels Spalthauttransplantation möglich erschien. Die auf den gesamten Defekt transplantierte Spalthaut heilte jedoch lediglich im proximalen Ausläufer des Defektes ein, während sich in den distalen zwei Dritteln des Defektes die Spalthaut in ihrer Gesamtheit beim ersten Verbandswechsel vom Wundbett wieder ablöste. Auf der offen liegenden Muskulatur war offenbar zu wenig Granulationsgewebe für eine Anheilung vorhanden. Zur Förderung der Wundgranulation führten wir daraufhin über 10 Tage eine Vakuumversiegelung des Oberschenkeldefektes durch.

Verwendet wurde ein offenporiger (Porengröße 0,2-1 mm) Polyvinylalkoholschaum (PVA-Schaum), in dem Drainagen eingebracht sind (Vacuseal plus ${ }^{\circledR}$ von Polymedics, Abb. 2). Die Vacusealplatten wurden von uns entsprechend dem Defekt ausgeschnitten und mit Hautklammern (Leukoclip ${ }^{\circledR}$ ) am Wundrand befestigt. Insgesamt sechs Drainageschläuche wurden transkutan ausgeleitet und über einen Drain-Mehrfachanschluss an eine Saugpumpe angeschlossen. Der Unterdruck betrug ca. 400 bis 300 mbar, entsprechend den Angaben in der Literatur, die einen Druck von 800 bis 200 mbar je nach Chronizität der Wunden empfiehlt [6]. Das gesamte Wundgebiet wurde anschließend mit einer transparenten, selbstklebenden, wasserdampfdurchlässigen, aber bakterienundurchlässigen Polyurethanfolie versiegelt (Joban $2^{\circledR}$ ). Die korrekte Versiegelungstechnik zeigte sich in einem konkaven Einziehen der Folie in die poröse Oberfläche des Schaumstoffs. Wir wechselten die komplette Versiegelung alle 3 bis 4 Tage.

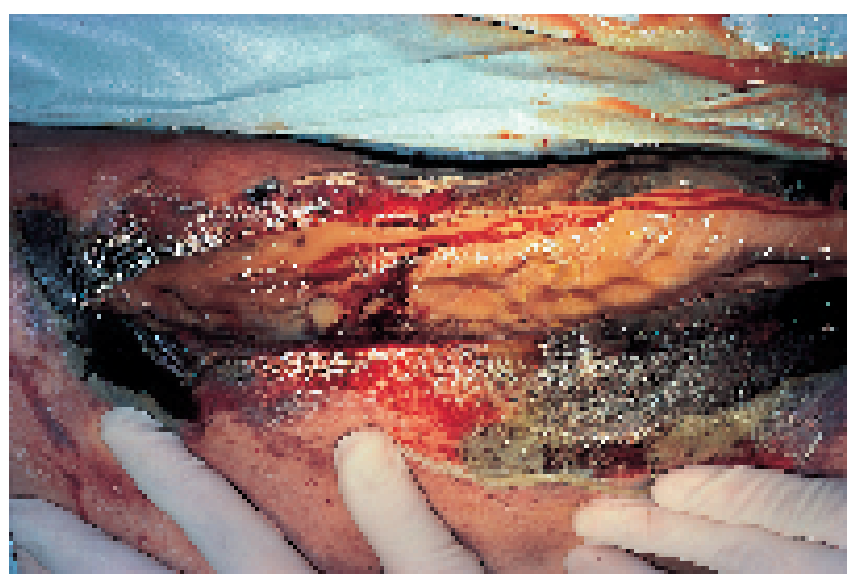

Abb.1 Großzügige Spaltung und ausgedehnte Nekrosektomie bei Fasciitis necroticans am rechten Oberschenkel eines 72-jährigen Patienten.

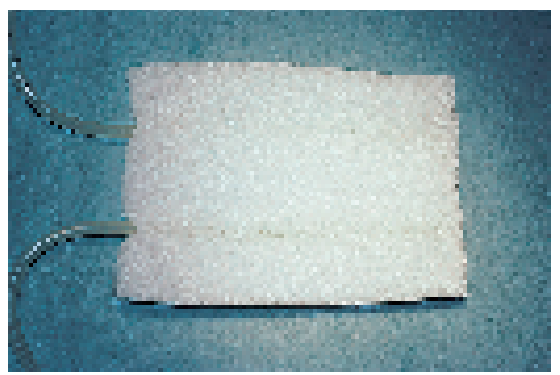

Abb. 2 Vacusealplatte mit zwei Drainageschläuchen.

Unter der Vakuumversiegelung bildete sich ein kräftiger Granulationsrasen aus, so dass der verbliebene Defekt ohne weitere Komplikation 10 Tage später mittels gemeshter Spalthaut erfolgreich gedeckt werden konnte (Abb. 3 u. 4).

\section{Diskussion}

Die Fasciitis necroticans ist eine seltene aber lebensbedrohliche Infektion des subkutanen Gewebes, welche mit einer Letalität von bis zu 73\% einhergeht [7,8]. Sie ist definiert als intensive Nekrose der oberflächlichen Faszie und fakultativ sekundär der Haut und selten der Muskulatur [9]. Gefürchtet werden eine rasch fortschreitende Ausdehnung der Phlegmone auf größere Gefäßnervenbündel und septische Komplikationen bis hin zum Multiorganversagen [10]. Aufgrund des Erregerspektrums wird die Erkrankung unterschieden in einen Typ I, der durch eine Mischinfektion von Anaerobiern, Enterobakterien oder Non-A-Streptokokken gekennzeichnet ist und einen Typ II der durch Streptokokken der Gruppe A verursacht wird und besonders foudroyant verläuft [7]. Unser Patient gehörte dem Typ I an, welcher eher zu subakuten Verläufen neigt [11]. Als prädisponierende Faktoren gelten Diabetes mellitus, generalisierte Arteriosklerose, maligne Grundleiden, Alkoholismus und iatrogene Suppression der Immunitätslage [10].

Nachdem bei unserem Patienten der Allgemeinzustand gebessert und ein sauberer Lokalbefund hergestellt worden waren, stellte sich die Frage nach einem geeigneten Verschluss des entstandenen Gewebedefektes. Ein knappes Drittel konnte durch Adaptationsnähte sowie Spalthauttransplantation ver- 


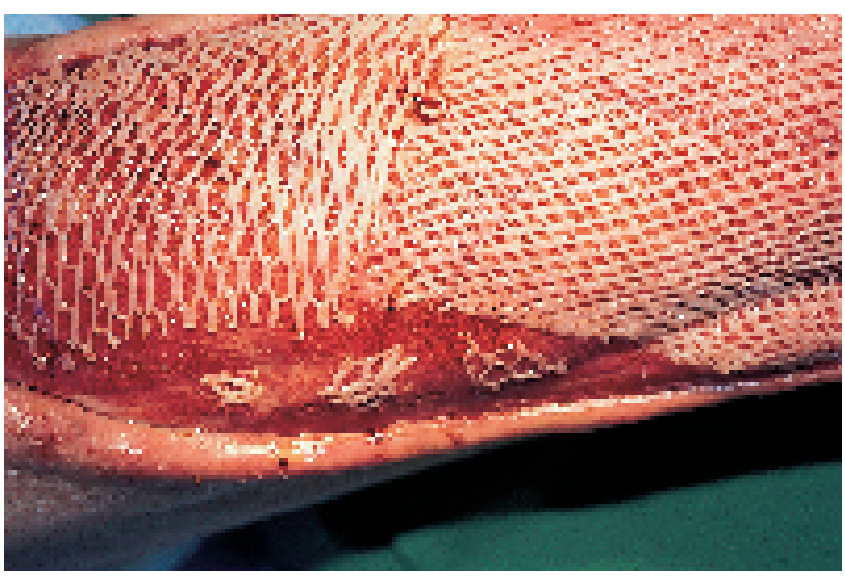

Abb.3 Nach zehntägiger Vakuumversiegelung hat sich gutes Granulationsgewebe im Defekt gebildet, wie im unteren Teil der Abbildung erkennbar. Unterschiedlich gemeshte Spalthaut wird aufgelegt.

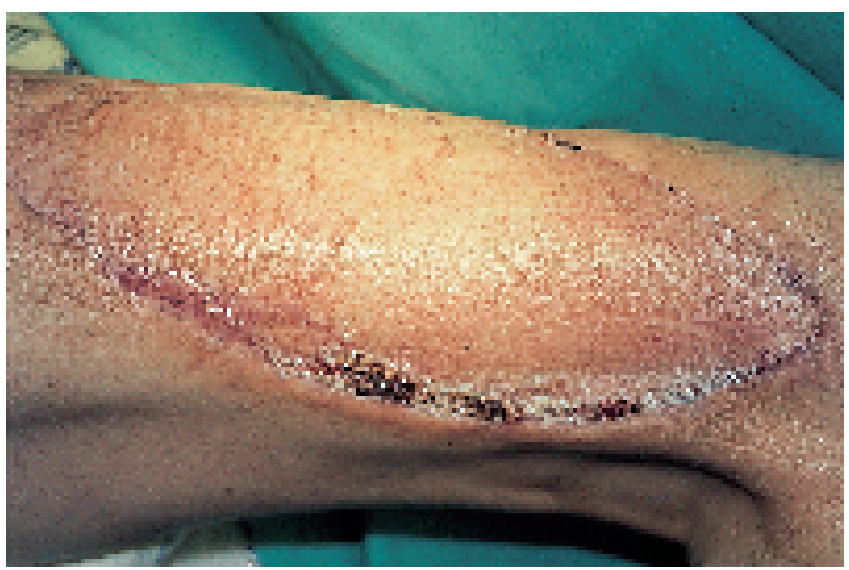

Abb.4 Problemloses Einheilen der Spalthaut auf dem durch Vakuumversiegelung vorbereiteten Wundgrund.

schlossen werden. Bei den restlichen zwei Dritteln war die erste Spalthauttransplantation wahrscheinlich mangels ausreichendem Granulationsgewebe erfolglos. Hier wurde die erstmals 1993 beschriebene Vakuumversiegelung angewandt.

Diese Versiegelung erscheint relativ einfach durchführbar und auch kostengünstig zu sein (DM 19,50 pro Vacusealplatte, DM 7,54 pro $35 \times 35 \mathrm{~cm}$ Polyurethanfolie). Eine geeignete Vakuumpumpe wird vielerorts bereits zur Verfügung stehen. Weitere Vorteile des Verfahrens sind der sichere Schutz der Wunde vor Superinfektion durch Hospitalkeime, ein vollständiges Evakuieren vom Wundsekret, eine deutliche Schmerzreduktion und die gute Mobilisierbarkeit des Patienten. Das Ergebnis besteht in der Bildung eines gleichmäßig strukturierten und gut vaskularisierten Granulationsrasens, welcher eine optimale Voraussetzung für das Einheilen von Spalthauttransplantationen bietet $[2,12]$.

In tierexperimentellen Untersuchungen an Schweinen konnten die positiven Wirkungen auf das Gewebe im Detail nachgewiesen werden. Im Wesentlichen bestehen sie in einem Anstieg des Blutflusses auf das Vierfache, einer Steigerung der Bildung von Granulationsgewebe um bis zu 103\% und einer
Reduktion der Bakterienzahl im Gewebe von anfänglich $10^{8} / \mathrm{g}$ Gewebe auf unter $10^{5} / \mathrm{g}$ Gewebe nach dem 4. Tag, was als Grenze für eine klinische Infektion angesehen wird [1].

In neueren Berichten wird zusätzlich auf die Möglichkeit der temporären Instillation von Antibiotika und von Antiseptika über das Trägersystem der Vakuumversiegelung hingewiesen. Dies bringe zusätzliche positive Effekte bei der Behandlung von akuten und chronischen Knochen- und Weichteilinfekten [6,13]. Ebenfalls positive Erfahrungen werden berichtet bei der Behandlung von Ulcera cruris mittels Kombination von moderner Varizenchirurgie (inklusive endoskopischer subfaszialer Diszision der Perforansvenen) und paratibialer Fasziotomie mit der Vakuumversiegelung [14].

Das genaue Indikationsgebiet der Vakuumversiegelung bedarf jedoch offensichtlich noch einer weiteren Klärung. Insbesondere stellt sich die Frage, ob und wann eine Vakuumversiegelung herkömmlichen Verfahren, z.B. einer Wundversorgung mit Polyurethanschaumverbänden (Syspur derm ${ }^{\circledR}$ ) entweder bezüglich der Bildung von Granulationsgewebe oder aber von der Kostenseite her überlegen ist. Hierzu gibt es unseres Wissens noch keine Studien. Ein direkter Vergleich, wenn möglich an den gleichen Patienten, wäre jedoch offensichtlich von großem Interesse.

\section{Danksagung}

Die Autoren danken Herrn Chefarzt Dr. Merbold (Unfallchirurgische Klinik, Vogtland-Klinikum Plauen GmbH) für seine Unterstützung bei der Einführung des Verfahrens der Vakuumversiegelung an unserer Klinik.

\section{Literatur}

${ }^{1}$ Morykwas MJ, Argenta LC, Shelton-Brown EI, McGuirt W. Vacuum-assisted closure: a new method for wound control and treatment: Animal studies and basic foundation. Ann plast Surg 1997; 38: $553-562$

2 Fleischmann W, Strecker W, Bombelli M, Kinzl L. Vakuumversiegelung zur Behandlung des Weichteilschadens bei offenen Frakturen. Unfallchirurg 1993; 96: 488-492

${ }^{3}$ Nuber V, Tilgen W, Dill-Müller D. Chronisches semizirkuläres Ulcus cruris - ein klinischer Verlauf unter Vakuumversiegelung. Z Hautkr 1999; 74: 535

${ }^{4}$ Armstrong DG, Wunderlich RP, Lavery LA. Reaching Closure with skin stretching. Clin podiat Med Surg 1998; 15: 109-116

${ }^{5}$ Caruso DM, King TJ, Tsujimura RB, Weiland DE, Schiller WR. Primary closure of fasciotomy incisions with a skin-stretching device in patients with burn and trauma. J Burn Care Rehab 1997; 18: $125-132$

${ }^{6}$ Fleischmann W, Russ M, Westhauser A, Stampehl M. Die Vakuumversiegelung als Trägersystem für eine gezielte lokale Medikamentenapplikation bei Wundinfektionen. Unfallchirurg 1998; 101: 649-654

${ }^{7}$ Kujath P, Eckmann C, Benecke P. Diagnose und Therapie der nekrotisierenden Fasziitis. Dtsch Med Wschr 1995; 120: 965-968

${ }^{8}$ Mundigler G, Geppert A, Henk C, Girsch W, Siostrzonek P. Extensive necrotizing fasciitis in a diabetic patient. Wien Klin Wschr 1998; 110: 446 - 448

${ }^{9}$ Bong J, Krawzak HW, Hohlbach G. Fasciitis necroticans. Z Dermatol 1996; 182: $138-143$

${ }^{10}$ Maron S, Pönnighaus JM, Kowalzick L. Selten aber wichtig: Fallbericht einer Fasciitis necroticans. Z Hautkr 1997; 72: 826-828 
${ }^{11}$ Imamura Y, Kudo Y, Ishii Y, Shibuya H, Takayasu S. A case of subacute necrotizing fasciitis. J Dermatol 1995; 22: 960-963

${ }^{12}$ Bauer P, Schmidt G, Partecke BD. Möglichkeiten der Vorbehandlung von infizierten Hautweichteildefekten durch Vakuumversiegelung mit PVA-Schaumstoff. Microchir Plast Chir 1998; 30: 20 30

13 Thöner B, Fleischmann W, Moch D. Wundbehandlung durch Vakuumversiegelung. Krankenpflege-Journal 1998; 36: 78-82

14 Thiele H, Kohler U. Das Ulcus cruris venosum. Langenbecks Arch Chir Supplement Kongreßband 1997; 114: 513-516
Dr. med. Albert Frank

Hautarztpraxis

Hochwartstraße 13

95643 Tirschenreuth 